\title{
Scintigraphic anatomy of coronary artery disease in digital thallium-201 myocardial images
}

\author{
R J WAINWRIGHT \\ From the Department of Cardiology and Department of Nuclear Medicine, Guy's Hospital, London
}

SUMMARY One hundred and eight patients with single and multiple vessel coronary artery disease confirmed by arteriography were evaluated by exercise thallium-201 $\left({ }^{201} \mathrm{Tl}\right)$ myocardial scintigraphy to determine the scintigraphic appearances of specific coronary stenoses. In general proximal stenoses caused more widespread, but not necessarily more severe, myocardial tracer deficit than distal stenoses. In particular, proximal dominant right coronary artery disease was specifically associated with extensive inferior wall tracer deficit in the anterior scintigram, whereas proximal left circumflex disease caused similar tracer depletion best visualised in the left lateral scintigram. A triad of uptake defects was caused by left anterior descending coronary artery disease: viz. apical tracer deficit (anterior view) in $71 \%$ of lesions, septal tracer deficit (left anterior oblique view) in $83 \%$ of lesions, and anterolateral wall tracer deficit (left lateral projection) in $72 \%$ of lesions. The last defect has been termed a 'diagonal window' because it was associated with independent disease of the main diagonal branch of the left anterior descending coronary artery or with disease in the main left anterior descending artery situated proximal to this branch. Diagonal window tracer deficit was the most useful scintigraphic sign distinguishing proximal from distal disease in the left anterior descending coronary artery. False negative scintigraphic defects occurred more commonly in patients with triple vessel disease and in association with well-developed coronary collateral vessels. Certain scintigraphic patterns of ${ }^{201} \mathrm{Tl}$ myocardial accumulation appear invaluable in the noninvasive localisation of stenoses within specific coronary arteries and thus may be useful in predicting life-threatening coronary artery disease which should be confirmed by definitive coronary arteriography. The digital ${ }^{201} \mathrm{Tl}$ myocardial scintigram also provides an independent functional guide to the interpretation of coronary arteriograms and may be helpful in the planning of aortocoronary bypass graft surgery.

Myocardial imaging with thallium-201 $\left({ }^{201} \mathrm{Tl}\right)$ has become a useful clinical technique in the evaluation of patients with regional myocardial ischaemia ${ }^{1-3}$ and myocardial infarction. ${ }^{45}$ The topography of tracer deficit evident in the ${ }^{201} \mathrm{Tl}$ scintigram is determined principally by the distribution of regional ischaemia and necrosis within the myocardium. Tracer deficit developing during exercise can be related to critical stenoses in specific coronary arteries and occurs in regions of the scintigram corresponding to the perfusion beds of these narrowed coronary vessels. ${ }^{6}$ The location and extent of specific coronary artery perfusion territories and their correlation with tracer

Received for publication 10 March 1981 deficit in the ${ }^{201} \mathrm{Tl}$ scintigram have previously been investigated by imaging the myocardium after labelled microspheres were selectively injected into the coronary circulation during arteriography. ${ }^{7}$ Despite such studies the features of exercise ${ }^{201} \mathrm{Tl}$ myocardial scintigrams which help to localise stenoses to specific coronary arteries are not well documented. In particular, there is little information about the scintigraphic appearances which may differentiate proximal coronary artery disease from distal disease. This study correlates the distribution of myocardial ${ }^{201} \mathrm{Tl}$ accumulated during exercise with the angiographic severity and location of disease in specific coronary arteries and attempts to identify scintigraphic patterns which may be useful in the prediction of individual coronary artery stenoses. 


\section{Patients and methods}

One hundred and eight patients (103 men and five women; mean age 51 years; range 28 to 64 years) with ischaemic heart disease documented by coronary arteriography were selected for exercise ${ }^{201} \mathrm{Tl}$ myocardial scintigraphy according to the avilability of tracer. The majority of patients were limited in their ordinary activities by angina pectoris despite adequate medical treatment 28 patients had single vessel disease, 38 patients had double vessel disease, 36 patients had triple vessel disease, and six patients had quadruple vessel disease. Two patients had significant stenosis of the main left coronary artery in addition to other coronary disease.

Patients with systemic or pulmonary hypertension, previous cardiac surgery, valvular and congenital heart disease were excluded from this study. Recent myocardial infarction (within three months) and unstable angina pectoris were further reasons for patient exclusion.

\section{MYOCARDIAL SCINTIGRAPHY}

Myocardial imaging with ${ }^{201} \mathrm{Tl}$ was performed within five days of coronary arteriography in all patients. No patient had clinical or electrocardiographic evidence of myocardial damage attributable to coronary arteriography. $1.5 \mathrm{mCi}$ of ${ }^{201} \mathrm{Tl}$ was administered intravenously while the patient performed graded maximal exercise on a Monark bicycle ergometer monitoring lead V5 of the electrocardiogram. Care was taken to maintain the end-point exercise level for approximately two minutes after ${ }^{201} \mathrm{Tl}$ had been administered. This manoeuvre enabled sufficient time for myocardial extraction and distribution of tracer to reflect accurately the altered regional perfusion prevailing at the limiting end-point of exercise. This end-point was angina pectoris in $80 \%$ of patients. Ten minutes after exercise, myocardial images were acquired in four projections, viz. anterior, left anterior oblique (LAO) $45^{\circ}$ and $55^{\circ}$, and a full left lateral view using an Ohio-Nuclear series 100 scintillation camera with a high-sensitivity parallelhole collimator. In each projection 200000 counts were acquired from the region of the myocardium using a $20 \%$ window centred on the $72.5 \mathrm{keV}$ mercury $x$-ray peak emitted by ${ }^{201} \mathrm{Tl}$ decay. A lead cape with a central hole was placed on the patient's chest, surrounding the heart and thus reducing the contribution of activity from regions other than the myocardium. Each image took approximately four to eight minutes to acquire and was simultaneously recorded on Kodak transparency film and magnetic disc in a $64 \times 64$ matrix after digital conversion using a dedicated minicomputer (DEC Gamma 11). In some cases delayed images of the myocardium were acquired four to six hours after the initial study. Informed consent was obtained from all patients and no complications occurred from exercise testing or ${ }^{201} \mathrm{Tl}$ myocardial imaging.

INTERPRETATION OF MYOCARDIAL IMAGES

The four digital images from each patient were enhanced by a $50 \%$ background erase, interpolated and displayed on a television monitor in 16 grades of colour which were linearly related to image intensity. Using an electronic light marker controlled by a joy-stick at the computer console each scintigram was subdivided into seven equal segments around its circumference. Each segment thus corresponded to approximately $14 \%$ of the total myocardial image. In addition, the anterior and left lateral projection of each patient were divided into equal superior and inferior areas about the long axis (base-apex) of the image. The ratio of tracer activity in these areas was computed and a normal range established.

The circumferential segments were expressed as average counts per digital matrix element and compared with a normal myocardial profile previously established from a store of ${ }^{201} \mathrm{Tl}$ scintigrams obtained from 22 patients with no evidence of cardiac disease and normal volunteers (Fig. 1). A typical normal ${ }^{201} \mathrm{Tl}$ myocardial scintigram is shown in Fig. 2. Significant myocardial tracer deficit was defined as segmental activity less than the $95 \%$ lower confidence interval.

Minimal image defects defined in this manner corresponded approxmately to a 12 to $15 \%$ regional change in count rate (four gradations of colour) estimated from the colour scale displayed beside the image. This quantitative technique was highly reproducible, the interobserver coefficient of variation being less than $1.5 \%$. The relation between quantitative and qualitative analysis of myocardial images was also examined. All scintigrams were reviewed independently by two experienced observers without knowledge of the coronary anatomy and their observations compared with quantitative segmental analysis. Excellent agreement was found between the two methods $(>90 \%)$ and interobserver agreement was similarly high. The few observer disagreements were resolved by subsequent review of the relevant scintigrams and consensus of a third observer was obtained if necessary. The scintigraphic data presented in this report are based upon the combined results of both analytical techniques.

CORONARY ARTERIOGRAPHY

Coronary arteriography was performed by either the Sones or Judkins technique. A significant stenosis was defined as a reduction of the lumen equal to or greater than $50 \%$ of the diameter in at least one coronary 
NORMAL COUNT PROFILE
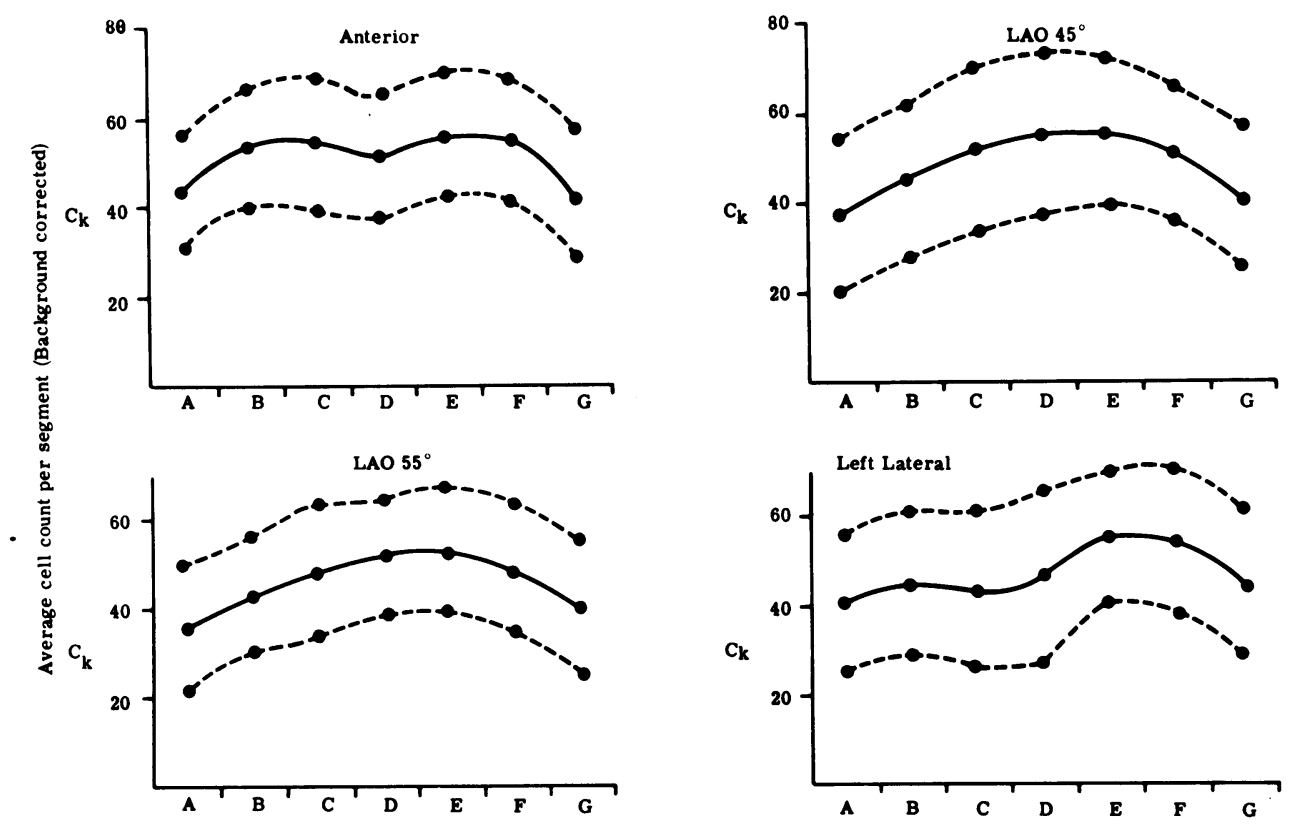

Fig. 1 Quantitative segmental myocardial profile in subjects with normal hearts. Dotted lines indicate $95 \%$ confidence limits.

artery. All coronary arteriograms were technically adequate and were reviewed independently by three observers in ignorance of the scintigraphic findings. Note was made of the site, severity, and number of significant coronary stenoses and coronary occlusions together with any associated coronary collateral channels. All observers agreed about complete occlusion of a vessel and there was greater than $90 \%$ agreement with regard to the site and significance of a stenosis. Interobserver variation in the assessment of coronary collateral development was small. ${ }^{8}$ Left ventricular function was also qualitatively assessed from a contrast left ventriculogram.

${ }^{201} \mathrm{Tl}$ myocardial scintigrams of patients with disease in a particular coronary artery were then compared with scintigrams from patients with disease in other coronary arteries, and with normal scintigrams.

Statistical comparison of the data was performed using the $\chi^{2}$ with Yates's correction or the Fisher exact test.

\section{Results}

FEATURES OF RIGHT CORONARY ARTERY DISEASE

Sixty-six patients had significant disease in the right coronary artery (RCA) either alone or combined with disease in other vessels. Sixty-two patients had a dominant right coronary artery, three patients a recessive right coronary artery, and one patient a pattern of mixed coronary dominance.

The left lateral projection was usually normal in patients with right coronary artery disease. In the anterior and left anterior oblique projections, however, three patterns of tracer distribution were apparent.

(i) Significant proximal disease in a dominant right coronary artery was characteristically associated with tracer reduction in the inferior wall of the left ventricle seen in both anterior and left anterior oblique projections (pattern i) Fig. 3a and b. This appearance was found in 22 out of $62(35 \%)$ patients. The majority of these patients $(15 / 22 ; 68 \%)$ had complete occlusion of the right coronary artery between its origin and the acute cardiac margin. The remaining patients all had severe stenoses in a similar location, that is the proximal one-third of the right coronary artery. Scintigraphic pattern i was never associated with right coronary artery disease solely confined to the distal one-third of its distribution. Two patients had isolated proximal occlusion of the right coronary artery alone; both developed typical pattern i tracer deficit in their scintigrams.

(ii) Nine patients manifested tracer deficit in the inferior wall of the left ventricle seen only in the 


\section{NORMAL THALLIUM 201 MYOCARDIAL IMAGES}

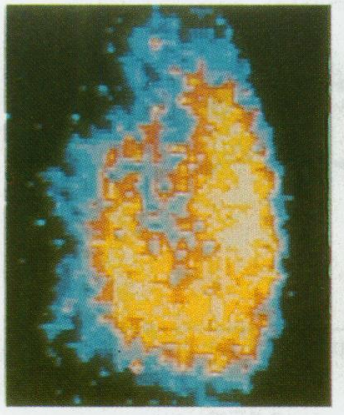

ANT

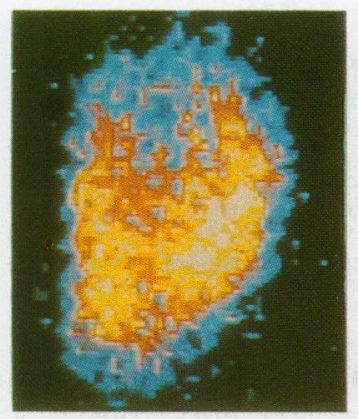

$\mathrm{LAO} 55^{\circ}$

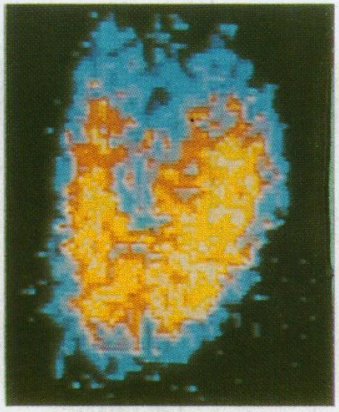

LAO $45^{\circ}$

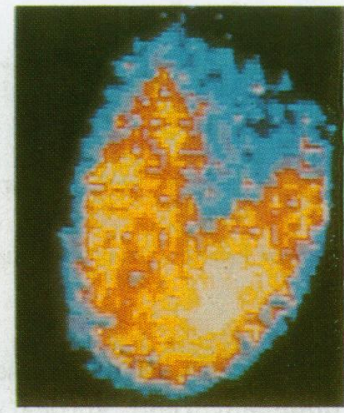

L LAT
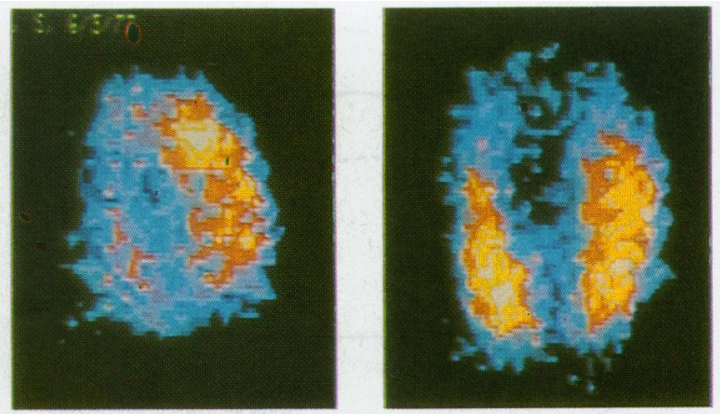

Case 1
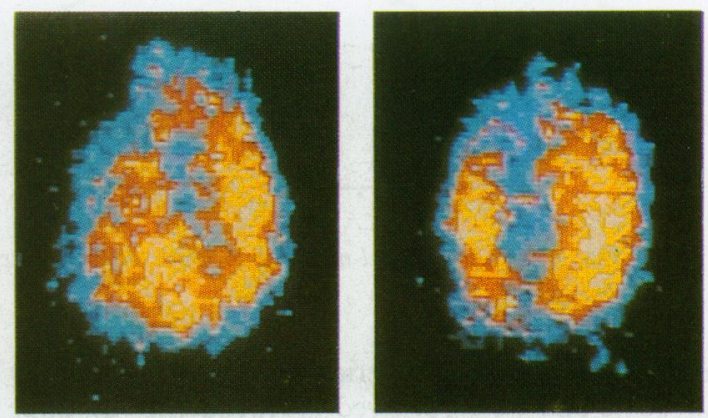

Case 2

Fig. 2 Normal appearances of a colour display digital ${ }^{201} \mathrm{Tl}$ scintigram in four standard projections.

anterior scintigram (pattern ii, Fig. 3b). Only three patients with this appearance had complete proximal occlusion of the right coronary artery compared with the 15 patients with pattern $i$ tracer deficit $(p<0.01)$. Moreover the majority of patients (seven) in this group had well-developed coronary collateral vessels compared with only 12 patients in the group with pattern i tracer deficit $(p<0.01)$. Only one patient had pattern ii tracer deficit without adequate development of distal right coronary artery collateral vessels. It was also interesting that no patient in this group had right coronary artery disease distal to the acute cardiac margin. Thus, inferior wall tracer deficit in the anterior view was specifically associated with proximal disease in a dominant right coronary artery.

(iii) Finally 18 patients had inferior wall tracer deficit seen in the left anterior oblique view but not in the anterior projection (pattern iii, Fig. 3a and b). There was no significant difference in the distribution of proximal and distal right coronary artery disease in

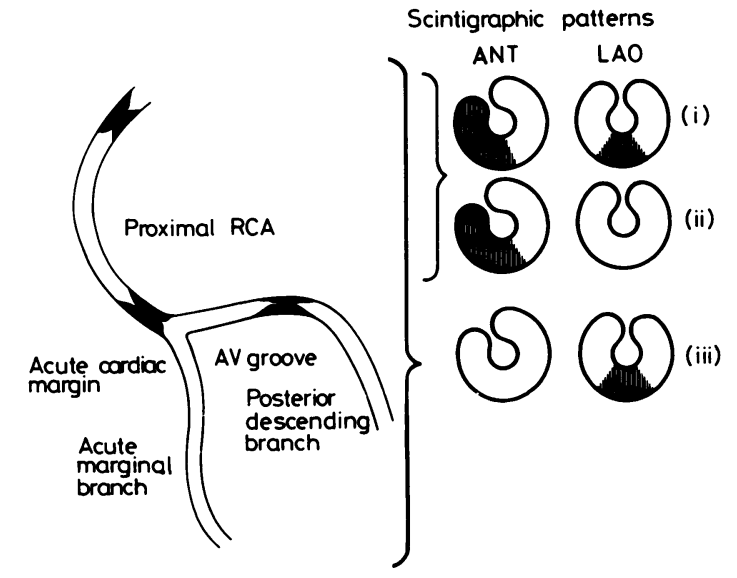

Fig. 3 (Above) Digital ${ }^{201} \mathrm{Tl}$ scintigrams of right coronary artery (RCA) disease. Case 1 had severe disease in the proximal RCA (dominant); case 2 had distal RCA disease. (Below) Summary of the scintigraphic patterns associated with right coronary artery disease. 
this group (11 patients v. seven patients). Complete proximal occlusion of the right coronary artery was found in seven patients, five of whom had welldeveloped distal coronary collateral vessels. One patient had complete proximal right coronary artery occlusion associated with large homocoronary collateral vessels which supplied a reconstituted arterial segment in the atrioventricular groove: the posterior descending artery however remained unopacified and reduced perfusion in this distal territory presumably accounted for the defect in the left anterior oblique scintigram. In another patient there was a large right ventricular branch of the right coronary artery arising just before the vessel became totally occluded. This branch was undiseased and particularly well visualised in the anterior projection during arteriography. Its presence may have contributed to the absence of tracer deficit in the anterior ${ }^{201} \mathrm{Tl}$ scintigram. The remaining four patients with proximal right coronary artery disease and pattern iii tracer deficit had only moderately severe coronary stenosis (50 to $70 \%$ narrowing).

It was interesting that the prevalence of retrograde coronary collateral vessels was greater in patients with pattern ii tracer deficit than with pattern $i(p<0.01)$ or with pattern iii $(p<0.05)$. Furthermore there was no significant difference in the prevalence of multiple vessel disease among these three scintigraphic patterns. Therefore, retrograde coronary collateral perfusion was probably an important factor determining the absence of inferior wall tracer deficit in the left anterior oblique scintigram of patients with severe right coronary artery disease.

Three patients with recessive right coronary artery disease had no apparent myocardial tracer deficit in the distribution of this vessel which presumably subtended a region too small for scintigraphic detection. One patient with mixed coronary dominance had right coronary artery disease without left circumflex (LCx) coronary disease and developed pattern iii tracer deficit in the myocardial scintigram.

Finally no patient with right coronary artery disease had isolated apical reduction of tracer in the anterior scintigram unless concomitant left anterior descending coronary artery disease was also present.

RIGHT CORONARY ARTERY DISEASE WITH NORMAL TRACER ACCUMULATION (FALSE NEGATIVES )

Thirteen patients (20\%) with dominant right coronary artery disease had completely normal tracer uptake in both anterior and left anterior oblique scintigraphic projections. Seven of these patients had excellent collateral filling of the distal right coronary artery bed. The remaining patients all had moderate disease (50 to $60 \%$ narrowing of the coronary lumen). None of these patients had single vessel right coronary artery disease, but concomitant multiple vessel disease was common (nine patients had triple vessel disease).

FALSE POSITIVE SCINTIGRAPHIC PATTERNS OF RIGHT CORONARY ARTERY DISEASE

Eleven out of 42 patients had tracer deficit associated with severe right coronary artery disease but coronary arteriography disclosed either no disease in this vessel or only mild disease (four patients) causing less than $50 \%$ narrowing of the coronary lumen. It is well known that coronary angiography may underestimate the severity of coronary disease when compared with necropsy findings. ${ }^{9} 10$ Tracer deficit seen in the scintigrams of patients with only mild atheroma may therefore reflect haemodynamically significant right coronary artery disease despite the more reassuring angiographic assessment. It is difficult to regard uptake defects in these patients as true false positives.

In the remaining seven patients with a normal appearance of the right coronary artery, inferior myocardial tracer accumulation seen in the anterior projection was also completely normal except in one patient with severe diffuse disease of the left circumflex coronary artery. This was the only patient in whom left circumflex coronary artery disease was associated with significant inferior wall tracer deficit in the anterior scintigram. Nevertheless, false positive inferior wall tracer deficit did occur in the left anterior oblique projection in some patients with severe concomitant left anterior descending coronary disease. Three patients had large apicoanterior ventricular aneurysms which caused distortion of the usual myocardial segmental relation. In patients without a left ventricular aneurysm false positive inferior wall tracer deficit was usually seen in only one left anterior oblique projection and was then clearly an extension of severe septal tracer deficit into the inferior wall caused by concomitant left anterior descending coronary artery disease. If this appearance is misinterpreted, however, the false positive rate remains as seven out of 42 patients (17\%). Finally, no patient with a normal right coronary artery had inferior wall tracer deficit apparent in both anterior and left anterior oblique scintigrams.

\section{FEATURES OF LEFT CIRCUMFLEX CORONARY}

ARTERY DISEASE

Seventy-one patients had significant disease in the left circumflex cornary artery which was usually associated with disease in other coronary vessels. Only two patients had single vessel left circumflex coronary artery disease. In five patients the left circumflex coronary artery had a dominant distribution. Fig. 4a and $b$ show typical patterns of tracer deficit attributable to disease in various parts of this coronary artery. 

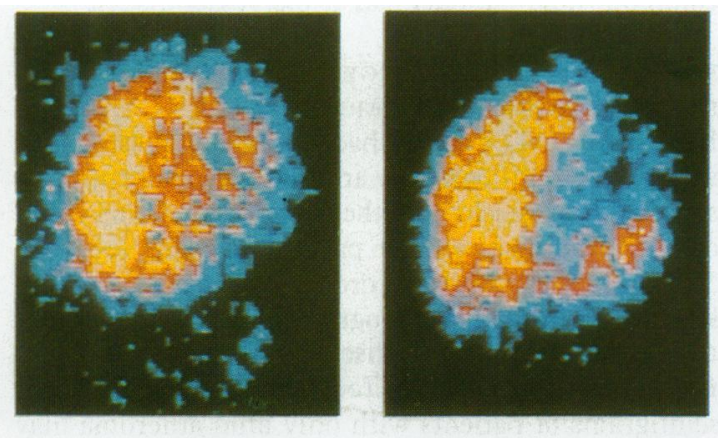

Case 1
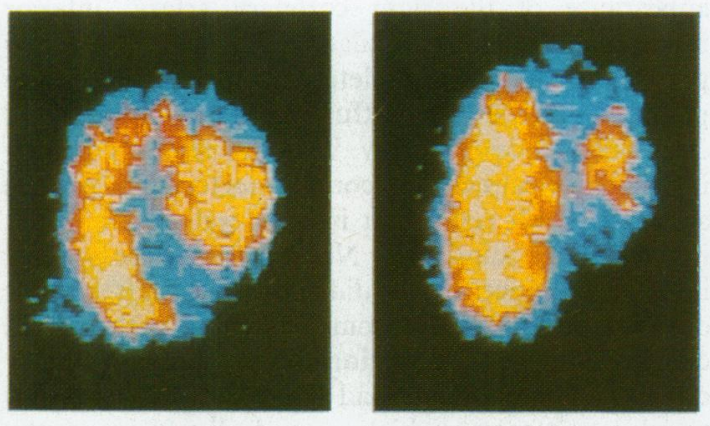

Case 2

The anterior scintigram was usually normal in patients with left circumflex coronary artery disease, but several different patterns were seen in the left anterior oblique and left lateral scintigram.

\section{LEFT CIRCUMFLEX DEFECTS IN TWO PROJECTIONS}

Twenty-five out of 71 (35\%) patients had tracer deficit apparent in both left anterior oblique and left lateral projections. Four patterns could be distinguished according to the size and position of the lesion (Fig. 4b).

(i) Pattern i occurred in 18 patients with disease in the main proximal portion of the left circumflex coronary artery. Most patients (15) had severe disease causing more than $80 \%$ narrowing of the coronary lumen.

(ii) Pattern ii was found in three patients, all of whom also had proximal left circumflex coronary artery

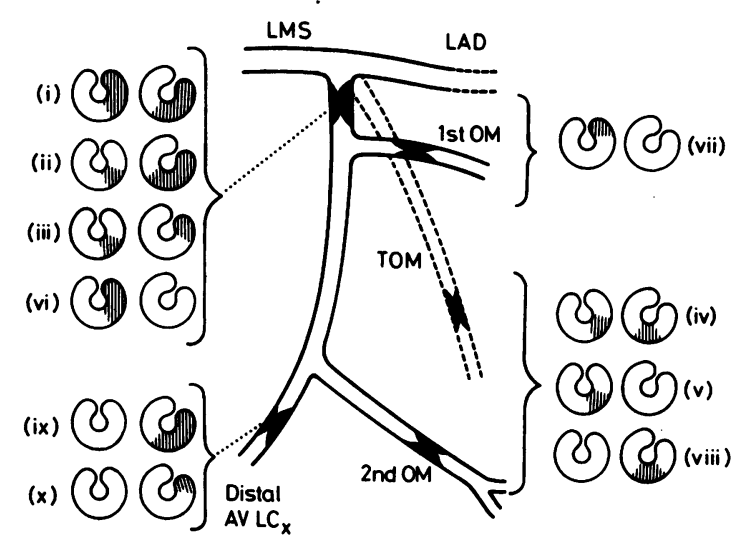

Fig. 4 (Left) Digital ${ }^{201} \mathrm{Tl}$ scintigram of left circumflex ( $\left.L C x\right)$ coronary artery disease. Case 1 had extensive posterolateral wall tracer deficit associated with proximal LCx coronary disease; case 2 had less extensive, discrete tracer deficit associated with distal LCx coronary disease. (Above) Summary of the scintigraphic patterns associated with left circumflex coronary artery disease. TOM, trifurcation obtuse marginal.

disease usually arising near the origin of the obtuse marginal branch.

(iii) Pattern iii was seen in two patients and also seemed to be a marker for proximal left circumflex coronary artery disease.

(iv) Pattern iv was characterised by discrete defects not extending to the basal portion of the scintigram and were seen in two patients both of whom had independent disease in the obtuse marginal branch but no proximal left circumflex coronary artery disease. This appearance therefore seemed to indicate disease in the distal portion of the left circumflex artery, specifically in the obtuse marginal branch.

LEFT CIRCUMFLEX ONE PROJECTION

(a) Left anterior oblique projection

Twenty-seven out of $71(38 \%)$ patients with left circumflex coronary artery disease had significant tracer deficit apparent only in the left anterior oblique 
projection. Three patterns were distinguishable according to the size of the defect in the posterolateral wall.

(i) Pattern $\mathrm{v}$ was seen in 13 patients with severe independent disease in the main obtuse marginal branch of the left circumflex coronary artery. The position of this defect in the posterolateral wall was specific for disease in the main obtuse marginal branch and it has been called an 'obtuse marginal window'. It was also seen in the left lateral projection (pattern viii).

(ii) Pattern vi occurred in four patients all of whom had severe disease in the main left circumflex coronary artery proximal to the obtuse marginal branch.

(iii) Six patients with proximal left circumflex coronary disease developed pattern vii tracer deficit which was also seen in four patients with disease in the first obtuse marginal artery.

\section{(b) Left lateral projection}

It was unusual to find tracer deficit associated with left circumflex coronary disease solely in the left lateral scintigram; only seven patients had this appearance. Three of these patients had discrete uptake defects near the cardiac apex (left lateral obtuse marginal window - pattern viii); all had severe disease of the obtuse marginal coronary artery. Two patients had tracer deficit extending throughout the inferolateral myocardial wall (pattern ix). Both patients had complete occlusion of the distal left circumflex artery including the obtuse marginal branch. The remaining two patients had discrete tracer deficit in the base of the inferolateral wall (pattern $\mathrm{x}$ ); both patients had complete occlusion of the left circumflex coronary artery in the atrioventricular groove.

\section{LEFT CIRCUMFLEX DISEASE WITH NORMAL TRACER UPTAKE (FALSE NEGATIVES)}

Twelve patients (17\%) with significant left circumflex coronary artery disease had completely normal tracer accumulation in both left anterior oblique and left lateral projections. Two patients in this group had well-developed collateral vessels filling the distal left circumflex territory retrogradely from the right coronary artery and six patients had proximal left circumflex coronary artery disease causing no more than $60 \%$ narrowing of the lumen. The remaining patients all had severe triple vessel disease, the left circumflex coronary artery being least affected.

FALSE POSITIVE SCINTIGRAPHIC PATTERNS OF LEFT CIRCUMFLEX CORONARY DISEASE

Five out of $37(14 \%)$ patients with a normal left circumflex coronary artery had disease in other coronary vessels but scintigrams falsely suggesting left circumflex disease. None of these patients, however, had tracer deficit in both the left anterior oblique and left lateral scintigram. Most patients developed isolated regions of tracer deficit situated in the basal posterolateral wall visualised in only one oblique view, the left anterior oblique $45^{\circ}$ rather than the left anterior oblique $55^{\circ}$. In contrast true positive left circumflex tracer deficit was detected more frequently in the left anterior oblique $55^{\circ}$ projection. One patient in this group did have proximal left circumflex disease causing 35 to $40 \%$ narrowing of the coronary lumen; another patient had a large left ventricular aneurysm which may have been relevant as previously discussed in the section on false positive right coronary artery tracer deficit.

In only one patient was false positive left circumflex tracer deficit detected solely in the left lateral projection. It was interesting that this patient had complete proximal occlusion of the right coronary artery associated with excellent retrograde collateral flow from a normal donor left circumflex coronary artery. During maximal exercise a coronary steal phenomenon may have developed between the left circumflex coronary artery and the right coronary artery thereby causing relative tracer deficit in the left circumflex arterial distribution despite its normal angiographic appearance.

TRIFURCATION OBTUSE MARGINAL DISEASE Myocardial tracer deficit produced by obtuse marginal coronary artery disease was similar in location regardless of whether the obtuse marginal artery arose directly from the main left coronary artery (trifurcation pattern) or arose in the more usual fashion as a branch of the left circumflex artery. Variation in the anatomy of coronary arteries obviously cannot be identified from analysis of a ${ }^{201} \mathrm{Tl}$ myocardial scintigram. Quadruple vessel disease affecting the right cornonary artery, left circumflex, left anterior descending, and trifurcation obtuse marginal coronary arteries therefore cannot be distinguished scintigraphically from triple vessel disease.

\section{FEATURES OF LEFT ANTERIOR DESCENDING}

CORONARY ARTERY DISEASE

Ninety-three patients had significant disease in the main left anterior descending coronary artery alone or combined with disease in other coronary vessels. A triad of characteristic scintigraphic defects was produced by disease in this vessel (Fig. 5). Furthermore, patients with disease in the proximal left anterior descending coronary artery had a different pattern of myocardial tracer distribution from patients with distal disease. 


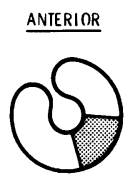

Apical deficit

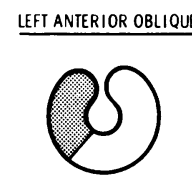

Septal deficit

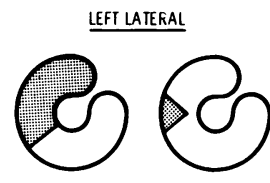

Diagonal window
Fig. 5 Scintigraphic patterns associated with left anterior descending coronary artery disease.

(1) Apical tracer deficit (anterior view)

Discrete apical tracer deficit, apparent only in the anterior scintigram, was caused by left anterior descending coronary disease in 66 out of $93(71 \%)$ patients (Fig. 5). The presence of this defect alone, however, did not indicate whether disease in the left anterior descending coronary artery was proximal or distal. Thus, there was no significant difference in the size or prevalence of apical tracer deficit in the scintigrams of patients with coronary disease situated before or after the first septal branch of the left anterior descending artery or with coronary disease in the main diagonal branch of the left anterior descending artery. Very extensive apical tracer deficit was more commonly found, however, with proximal left anterior descending coronary disease. Thus, apical tracer deficit occupying more than $40 \%$ of the whole myocardial image was three times more likely to be associated with proximal rather than distal left anterior descending coronary disease.

Fourteen of 15 patients with coronary disease sparing the left anterior descending artery had normal apical accumulation of tracer in the anterior scintigram. One false positive defect was seen in a patient with left circumflex coronary disease which produced severe apicolateral tracer deficit more clearly visualised in the left anterior oblique and left lateral scintigram.

Apical tracer deficit was not seen in 27 patients despite the presence of severe left anterior descending coronary artery disease. It was interesting that most of these false negative scintigrams (25) occurred in a subset of 28 patients with excellent calibre of the left anterior descending arterial lumen distal to the site of the coronary stenosis (Fig. 6).

\section{(2) Septal tracer deficit (left anterior oblique)}

This scintigraphic defect was slightly more useful than the apical tracer deficit in distinguishing proximal from distal stenoses and was found in 77 out of 93 patients $(83 \%)$ with left anterior descending coronary artery disease (Fig. 5). Fifty-two out of 56 (93\%) patients with left anterior descending disease situated proximal to the first septal artery developed

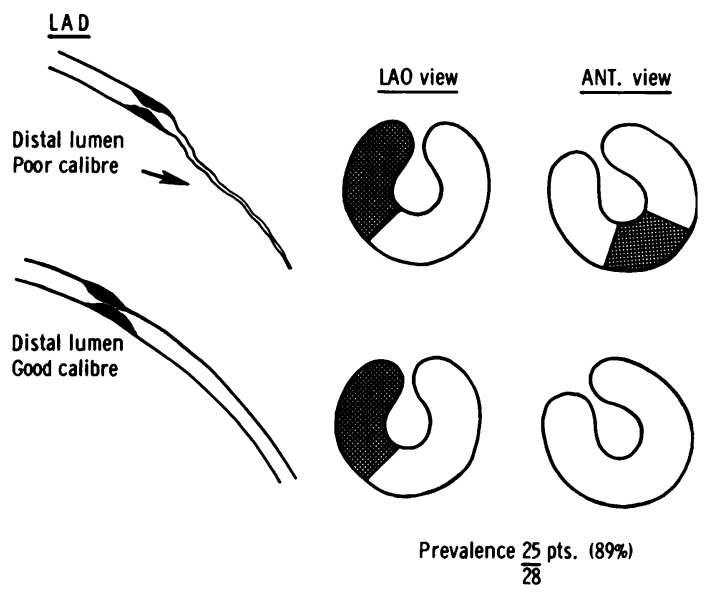

Fig. 6 Absent apical tracer deficit in the presence of proximal left anterior descending coronary artery disease is frequently associated with a good calibre lumen of the distal vessel.

significant septal tracer deficit compared with 25 out of $37(68 \%)$ with disease distal to this branch $(\mathrm{p}<0.05)$. In contrast there was no significant difference in the prevalence of septal tracer deficit when disease situated between the first septal and main diagonal branch was compared with more distal disease. Thus, the site of left anterior descending coronary artery disease with regard to the first septal artery, not the main diagonal artery, was most relevant to the prevalence of significant tracer deficit developing within the ventricular septum. Though pre-first septal left anterior descending coronary artery disease was more commonly associated with septal tracer deficit it was not possible to predict accurately the location of a stenosis in individual patients from this scintigraphic appearance alone.

The most severe examples of septal tracer deficit were usually associated with greater than $90 \%$ stenosis of the proximal left anterior descending coronary artery but in general the paucity of tracer accumulation within the septum was a poor guide to the longitudinal localisation of a stenosis.

In comparison, 13 out of 15 patients with atheroma not involving the left anterior descending coronary artery had completely normal tracer accumulation within the ventricular septum. Two patients with false positive septal tracer deficit had poor left ventricular function and an enlarged heart which may have contributed to diminished septal tracer activity through geometrical distortion and increased tracer attenuation.

Several patients with hypertrophy of the first septal artery had normal septal accumulation of tracer 
despite severe post-septal left anterior descending coronary disease. In these patients the first septal artery was clearly the largest vessel supplying the ventricular septum and presumably accounted for preservation of tracer uptake.

\section{(3) Diagonal window tracer deficit (left lateral view)}

In the left lateral projection tracer deficit attributable to left anterior descending coronary disease was commonly seen in the anterolateral wall of the scintigram, a region supplied by the diagonal branches of the left anterior descending coronary artery. Fig. 5 illustrates a small wedge defect seen in the upper half of the cardiac apex which has been called a 'diagonal window' because it appeared to be associated specifically with independent disease of the left anterior descending diagonal artery or with disease in the main left anterior descending coronary artery situated proximal to this branch. This defect often appeared as a discrete island of tracer attenuation lying within the myocardial border. Patients with complete proximal occlusion of the left anterior descending artery had extensive anterolateral tracer deficit extending from base to apex forming a massive window.

Diagonal window tracer deficit was found in 59 out of $82(72 \%)$ patients with coronary disease situated proximal to the left anterior descending diagonal branch compared with only two out of $11(18 \%)$ patients with disease distal to this branch $(p<0.001)$. In contrast no difference in the prevalence of a diagonal window occurred in patients with left anterior descending coronary disease found before the first septal artery compared with patients with disease distal to this branch, that is diagonal window tracer deficit per se did not distinguish pre-first septal from post-first septal left anterior descending coronary disease. It was equally apparent that ischaemia in the territory of the first septal artery was irrelevant to the prevalence of this defect and therefore unlikely to account for it. In comparison to apical tracer deficit (anterior view) or septal tracer deficit (left anterior oblique view) the presence of a diagonal window was the best scintigraphic discriminant of proximal and distal disease in the left anterior descending coronary artery. Fig. 7 summarises the scintigraphic anatomy relevant to the longitudinal localisation of left anterior descending coronary artery disease.

\section{PATIENTS WITH LEFT MAIN CORONARY ARTERY} STENOSIS

Only two patients had stenosis of the left main coronary artery in addition to other coronary disease. One patient had severe left anterior descending coronary artery disease proximal to the diagonal branch; the other patient had disease distal to this

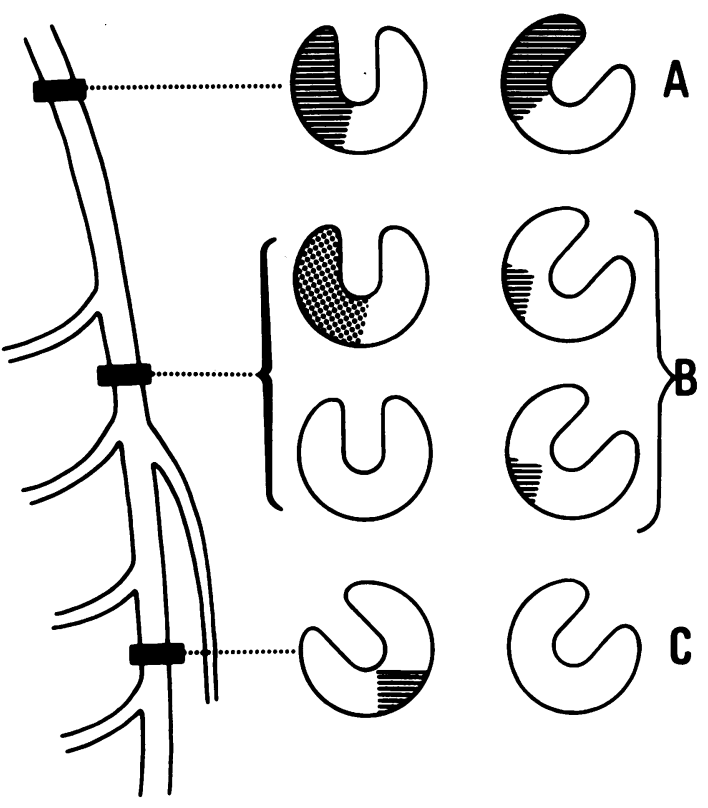

Fig. 7 Scintigraphic patterns in the longitudinal localisation of left anterior descending (LAD) coronary artery disease. Severe proximal $L A D$ coronary disease caused extensive septal and anterolateral wall tracer deficit (pattern A); LAD disease situated after the first septal artery and before the main diagonal branch caused less severe and sometimes absent septal tracer deficit but usually a diagonal window (pattern B); distal LAD disease usually produced apical tracer deficit in the anterior view only, with normal left anterior oblique and left lateral projections (pattern C).

branch. Interestingly, both patients developed positive diagonal window tracer deficit in the left lateral scintigram.

\section{COMBINATIONS OF TRACER DEFICIT IN}

MYOCARDIAL SCINTIGRAM

Tracer deficit produced by disease in individual coronary arteries may be combined in several characteristic patterns in the scintigrams of patients with multiple vessel coronary disease.

In general, right coronary artery disease was manifest by abnormal anterior and left anterior oblique scintigrams whereas left circumflex disease caused abnormal left anterior oblique and left lateral scintigrams. A normal left lateral scintigram was found in all 12 patients in this series with right coronary artery disease but no left circumflex coronary disease. Conversely in 17 out of 18 patients with left circumflex coronary disease but no evidence of right coronary artery disease the anterior scintigram was correspondingly normal. Thus, inferior wall tracer deficit seen in the anterior scintigram but not in 
the left lateral projection was a fairly specific marker for proximal disease in a dominant right coronary artery.

Fourteen out of $52(27 \%)$ patients with right coronary artery and left circumflex disease had inferior wall tracer deficit in both the anterior and left lateral projection confirmed by measuring an inferior/ superior wall activity ratio (normal ratios: anterior view $0.95 \pm 1 \cdot 13$; left lateral view $0.94 \pm 1 \cdot 14$ ). Conversely, 16 patients with this combination of disease had a normal inferior/superior wall activity ratio in both anterior and left lateral projections, but did have abnormal left anterior oblique scintigrams allowing correct identification of disease in one or both arterial systems. The remaining 12 patients with combined right coronary artery and left circumflex coronary disease had reduced inferior wall tracer accumulation in either the anterior or left lateral scintigram.

Finally, patients with triple vessel disease frequently showed evidence of impaired tracer accumulation in all three coronary territories of the left ventricle best visualised in the left anterior oblique scintigram (Fig. 8).

COMBINED DIAGONAL AND OBTUSE MARGINAL WINDOWS

In the left lateral scintigram of four patients in this series there was an extensive apical defect best described as combined obtuse marginal and diagonal window tracer deficit. Fig. 9 shows an example of this appearance in a patient whose coronary arteriogram confirmed severe disease in the obtuse marginal coronary artery and left anterior descending artery before the first diagonal branch. The myocardial perfusion beds of the diagonal left anterior descending artery and obtuse marginal artery appear to be juxtaposed at the periphery; thus severe disease in both branches produces a larger 'window' than disease in either vessel alone. It is important to recognise this single extensive defect at the cardiac apex which straddles the long axis of the myocardial image for it crosses the watershed of the left coronary branches and therefore suggests disease involving both major branches of the main left coronary artery. False negative interpretation for either left coronary artery branch may then be avoided.

\section{Discussion}

Conventional noninvasive methods of assessment often fail to provide the reassurance needed to deal with patients who may have lethal and unpredictable disease yet in whom coronary arteriography is an unattractive immediate investigation with expense, inconvenience and hazard hard to reconcile with the

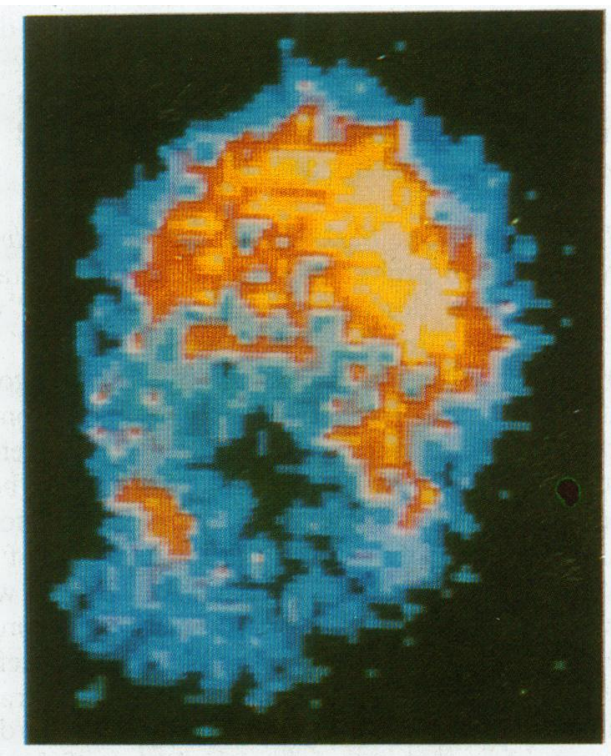

Fig. 8 Typical scintigraphic appearance of triple vessel coronary artery disease in the left anterior oblique view. Uptake defects extend into perfusion territories of all three major coronary arteries.

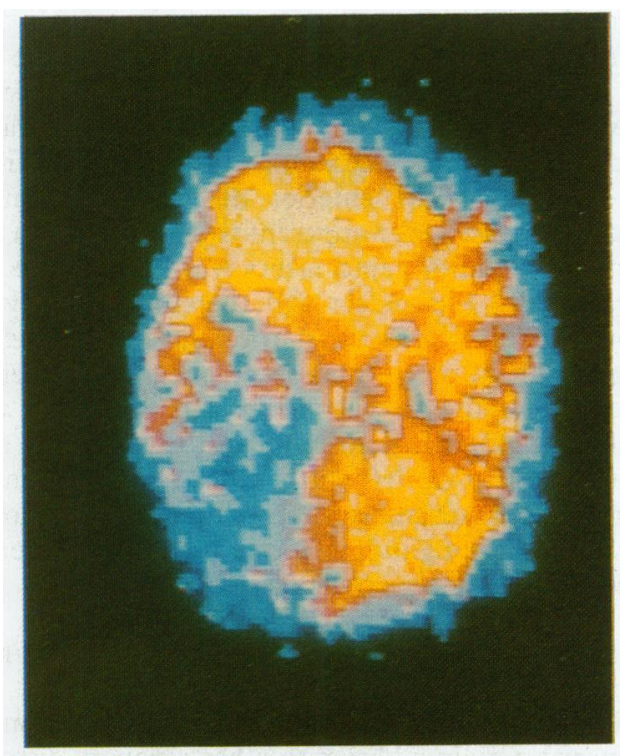

Fig. 9 Combined diagonal window and obtuse marginal tracer deficit appearing as a joint defect at the cardiac apex in the left lateral projection. 
magnitude of the clinical problem. This dilemma is encountered in the management of the following groups: asymptomatic patients with positive exercise electrocardiograms or a bad family history of ischaemic heart disease, young patients with recent inferior myocardial infarction and patients with atypical chest pain. Detection and localisation of coronary artery disease using a minimally invasive method such as exercise ${ }^{201} \mathrm{Tl}$ myocardial scintigraphy would provide an attractive alternative technique to indiscriminate coronary arteriography in the management of these patients. Disease associated with a high risk of sudden death or myocardial infarction would have a high probability of detection and could be confirmed by coronary arteriography. Conversely a normal scintigram or tracer deficit associated with less lethal coronary disease provides a measure of reassurance which may allow many patients to forego diagnostic arteriography.

This study indicates that stenoses causing greater than $50 \%$ narrowing of the coronary lumen produce characteristic defects of tracer accumulation in exercise ${ }^{201} \mathrm{Tl}$ myocardial scintigrams which allow disease to be attributed to specific coronary arteries including third generation vessels. It seems possible to assess whether proximal coronary artery disease is more likely than distal disease by analysing carefully the topography of tracer deficit found within the myocardium. Thus, proximal disease of a dominant right coronary artery could be predicted from inferior wall tracer deficit seen in both anterior and left anterior oblique projections; disease in a dominant left circumflex vessel only rarely accounted for this appearance. Profound tracer deficit in this distribution usually indicated complete occlusion in the proximal segment of the right coronary artery. In contrast, inferior wall tracer deficit visualised only in the left anterior oblique scintigram was attributable to distal right coronary artery disease or proximal right coronary artery disease with well-developed coronary collateral vessels. Failure to detect significant right coronary artery disease commonly occurred in the presence of well-developed coronary collateral vessels (both homocoronary and heterocoronary), severe concomitant multiple vessel disease, and disease in a recessive vessel.

Falsely positive right coronary artery tracer deficit was never seen in both anterior and left anterior oblique scintigrams. Severe concomitant left anterior descending coronary artery disease causing extensive septal tracer deficit, however, occasionally produced false positive inferior wall tracer deficit in the left anterior oblique view. In addition a dilated or aneurysmal left ventricle was frequently associated with false positive tracer deficit in the distribtuion of all coronary arteries.
Similar analysis of the scintigraphic features found with left circumflex coronary artery disease suggests that proximal stenosis of this vessel can also be predicted. In the left lateral scintigram all patients with inferior wall tracer deficit extending from apex to base had proximal left circumflex disease, a pattern analogous to that seen in the anterior scintigram of patients with proximal right coronary artery disease. Indeed, inferior wall tracer deficit visualised in both anterior and left lateral projections strongly suggested proximal disease in both right coronary artery and left circumflex coronary arteries. Conversely, distal left circumflex coronary disease, particularly involving the obtuse marginal artery, was usually associated with smaller, discrete areas of tracer deficit in the posterolateral wall which did not extend to the base of the heart.

Perhaps the most significant finding in this study was that proximal left anterior descending coronary disease could be detected with a high degree of confidence from the appearance of diagonal window tracer deficit in the left lateral scintigram. Of patients with left anterior descending coronary artery disease proximal to or within the main diagonal branch, $72 \%$ developed this scintigraphic defect compared with only $18 \%$ of patients with distal disease. Though slightly less sensitive than septal tracer deficit as a marker for left anterior descending coronary disease, diagonal window tracer deficit was quite a specific sign of proximal disease in the left anterior descending coronary artery and interestingly occurred in the two patients with left main coronary stenosis. This important defect was detected only in the full left lateral projection which should be a standard scintigraphic projection for ${ }^{201} \mathrm{Tl}$ myocardial imaging. This view was performed in the right lateral decubitus position, the patient lying across a stiff pillow to eliminate lateral thoracic curvature to the left, thus permitting the scintillation camera close access to the chest wall which is normally prevented by the prominence of the pelvic crest.

Further experience with increased numbers of patients is needed before the clinical value of diagonal window tracer deficit in the detection of patients with left main coronary artery disease can be determined, but there is no scintigraphic marker specific for coronary disease in this site. Diagonal window tracer deficit was usually associated with disease in the left anterior descending coronary artery situated between the ostium and the first diagonal artery, or with separate disease in the main diagonal artery itself. This scintigraphic information may be extremely useful in patients about to undergo aortocoronary bypass graft surgery, for independent diagonal branch left anterior descending coronary disease, which occasionally may be difficult to evaluate by con- 
ventional angiography, frequently merits a separate vein graft. ${ }^{11}$ In this context similar considerations apply to the value of absent apical tracer deficit in the anterior view: the absence of this defect in patients with known left anterior descending coronary artery disease was nearly always associated with excellent calibre of the distal left anterior descending coronary lumen. The appearance of normal tracer accumulation at the cardiac apex should, therefore, have excellent predictive accuracy for good distal run-off from a left anterior descending graft and thus for long term left anterior descending graft patency.

Some authors ${ }^{6}$ regard apical tracer deficit as a non-specific appearance commonly seen in normal hearts and have accordingly rejected this region from further consideration. This is a difficult area for interpretation because of normal apical thinning of the myocardium ${ }^{12}$; nevertheless in this study unequivocal apical tracer deficit confirmed by quantification was specifically associated with left anterior descending coronary artery disease. Furthermore this may be the only region in which left anterior descending coronary disease is manifest, particularly if stenoses are situated distal to the left anterior descending septal and diagonal branches. Thus, routine rejection of this important region may needlessly reduce the detection sensitivity of left anterior descending coronary artery disease.

Another dimension in the evaluation of ischaemic heart disease is provided by exercise ${ }^{201} \mathrm{Tl}$ myocardial scintigraphy which this study indicates is a promising technique in the detection and localisation of disease in specific coronary arteries, particularly disease affecting the left coronary system. The scintigraphic signs described in this report may be extremely useful in predicting coronary atheroma and selecting or excluding patients for definitive coronary arteriography. The planning of aortocoronary bypass graft surgery may be greatly facilitated, particularly when disease is suspected in the diagonal branch of the left anterior descending coronary artery. Finally the ${ }^{201} \mathrm{Tl}$ myocardial scintigram provides a valuable independent guide to the interpretation of the coronary arteriogram.

It remains to be emphasised that not all coronary artery disease can be reliably predicted in every patient entirely from analysis of tracer deficit found in $a^{201} \mathrm{Tl}$ myocardial scintigram. Perhaps the intention should be not so much to predict specific coronary artery disease, useful as that may be, but rather to describe common associations of coronary disease and myocardial tracer deficit so that gross disparities in individual patients can be explored more carefully.

Although stenoses can be accurately localised by coronary arteriography, this technique provides little information about the size of the myocardial perfusion territory jeopardised by ischaemia. ${ }^{201} \mathrm{Tl}$ myocardial scintigraphy gives direct information about this variable and may prove to be as useful as angiography in guiding prognosis.

Dr R J Wainwright was supported by a British Heart Foundation grant. This work has been submitted as part of a London M D Thesis.

\section{References}

1 Bailey IK, Griffith LSC, Rouleau J, Strauss HW, Pitt B. Thallium-201 myocardial perfusion imaging at rest and during exercise.Comparative sensitivity to electrocardiography in coronary artery disease. Circulation 1977; 55: 79-87.

2 Ritchie JL, Trobaugh GB, Hamilton GW, et al. Myocardial imaging with thallium-201 at rest and during exercise. Comparison with coronary arteriography and resting and stress electrocardiography. Circulation 1977; 56: $66-71$.

3 Wainwright RJ, Maisey MN, Curry PVL, Sowton E. Localisation of coronary artery disease by thallium-201 stress myocardial scintigraphy (abstract). $\mathrm{Br}$ Heart $\mathcal{f}$ 1978; 40: 1063-4.

4 Wackers FJT, Becker AE, Samson G, et al. Location and size of acute transmural myocardial infarction estimated from thallium-201 scintiscans. A clinicopathological study. Circulation 1977; 56: 72-8.

5 Hamilton GW, Trobaugh GB, Ritchie JL, Williams DL, Weaver WD, Gould KL. Myocardial imaging with intravenously injected thallium-201 in patients with suspected coronary artery disease. Analysis of technique and correlation with electrocardiographic, coronary anatomic and ventriculographic findings. Am 7 Cardiol 1977; 39: 347-54.

6 Lenaers A, Block P, Thiel EV, et al. Segmental analysis of Tl-201 stress myocardial scintigraphy. $7 \mathrm{Nucl}$ Med 1977; 18: 509-16.

7 Ritchie JL, Hamilton GW, Gould KL, Allen D, Kennedy JW, Hammermeister KE. Myocardial imaging with indium- $113 \mathrm{~m}$ and technetium-99m macroaggregated albumin. Am $\mathcal{F}$ Cardiol 1975; 35: 380-9.

8 Wainwright RJ, Maisey MN, Edwards AC, Sowton E. Functional significance of coronary collateral circulation during dynamic exercise evaluated by thallium-201 myocardial scintigraphy. Br Heart $\mathcal{F}$ 1980; 43: 47-55.

9 Schwartz JN, Kong Y, Hackel DB, Bartel AG. Comparison of angiographic and postmortem findings in patients with coronary artery disease. Am $\mathcal{F}$ Cardiol 1975; 36: $174-8$ 
10 Hutchins GM, Bulkley BH, Ridolfi RL, Griffith LSC, Lohr FT, Piasio MA. Correlation of coronary arteriograms and left ventriculograms with post mortem studies. Circulation 1977; 56: 32-7.

11 Wainwright RJ, Brennand-Roper DA, Maisey MN, Sowton E. Exercise thallium-201 myocardial scintigraphy in the follow-up of aortocoronary by-pass graft surgery. Br Heart F 1980; 43: 56-66.

12 Bradfield JWB, Beck G, Vecht RJ. Left ventricular apical thin point. Br Heart $\mathcal{F}$ 1977; 39: 806-9.

Requests for reprints to $\operatorname{Dr} R \mathbf{J}$ Wainwright, Cardiology Department, Guy's Hospital, London SE1 9RT. 